\title{
MINIMIZATION PROBLEMS FOR NONCOERCIVE FUNCTIONALS SUBJECT TO CONSTRAINTS
}

\author{
VY KHOI LE AND KLAUS SCHMITT
}

\begin{abstract}
We consider noncoercive functionals on a reflexive Banach space and establish minimization theorems for such functionals on smooth constraint manifolds. These results in turn yield critical point theorems for certain classes of homogeneous functionals. Several applications to the study of boundary value problems for quasilinear elliptic equations are included.
\end{abstract}

\section{INTRODUCTION}

Let $V$ be a reflexive Banach space and let

$$
F: V \rightarrow \mathbb{R}
$$

be a weakly lower semicontinuous functional and let $S \subset V$ be a weakly closed set. This paper is devoted to the problem of establishing the existence of points $u \in S$ such that

$$
F(u)=\min _{v \in S} F(v),
$$

and consequences of such results. If it is the case that $F$ is coercive, then classical results ([MW], [S]) establish a solution of this problem. The functionals considered here have the property that the perturbed functionals

$$
F_{\epsilon}(u)=F(u)+\epsilon\|u\|^{2},
$$

where $\|\cdot\|$ is the norm of $V$, are coercive for $\epsilon>0$ and that the solution sets $\left\{u_{\epsilon}\right\}$, of

$$
F_{\epsilon}(u)=\min _{v \in S} F_{\epsilon}(v)
$$

are a priori bounded. Solutions of the original problem are thus obtained via a limit process, letting $\epsilon \rightarrow 0$ (since $V$ is reflexive, bounded subsets have weakly convergent subsequences). This procedure (of elliptic regularization) has been widely used (see, e.g., [KS], [ASV1-3]); we here provide a class of functionals (and compatible manifolds $S$ ) for which this method of attack is applicable and include a variety of consequences such as critical point theorems (e.g., the

Received by the editors July 11, 1994 and, in revised form, January 9, 1995.

1991 Mathematics Subject Classification. Primary 35J15, 35J85, $49 \mathrm{~J} 40$.

Key words and phrases. Minimization of functionals, critical point theorems, noncoercive functionals, nonlinear boundary value problems, $p$-Laplacian.

Research supported by a grant from NSF. 
recent results in [BTW]) and various applications to boundary value problems for nonlinear elliptic partial differential equations.

The paper is organized as follows: In sections 2-3 we consider the case that $V=H$ is a Hilbert space, since matters are more transparent and easier to discuss. In section 2 we define a class of functionals (functionals having property $(P))$ for which the above described procedure yields a solution of (1.1) and we state and prove our main abstract existence theorem (Theorem 2.5). We further provide several (more easily verifiable) criteria which imply this property, and also criteria which describe manifolds $S$ which satisfy the necessary compatibility conditions. In section 3 we derive several critical point theorems (and several special cases of Theorem 2.5) which may be derived from Theorem 2.5 and Liusternik's theorem on Lagrange multipliers. The subsequent sections (4 and 5) are devoted to the more general situation where $V$ is a reflexive Banach space and extensions of the results in the earlier sections. Some of the analysis will be similar; when this is the case we shall so indicate and forgo the details; however, as we shall see, enough significant differences exist to warrant these sections. We conclude with several examples giving applications of the results established to the theory of boundary value problems for semilinear and nonlinear elliptic equations, including problems involving the $p$-Laplacian.

\section{General Results}

2.1 Assumptions and notations. Let $V=H$ be a Hilbert space with norm $\|\cdot\|$ and inner product $\langle\cdot, \cdot\rangle$. Let

$$
A: H \rightarrow H\left(=H^{*}\right)
$$

be a mapping such that the functional

$$
\varphi: H \rightarrow \mathbb{R}
$$

given by

$$
u \mapsto\langle A u, u\rangle
$$

is weakly lower semicontinuous. Let

$$
j: H \rightarrow \mathbb{R} \cup\{+\infty\}(\not \equiv \infty)
$$

be a convex, lower semicontinuous (hence weakly lower semicontinuous) proper functional such that (without loss in generality) $j(0)=0$.

Let $Y$ be a Banach space with norm $\|\cdot\|_{Y}$ and suppose that

$$
\psi: H \rightarrow Y
$$

is a completely continuous mapping. Let $\gamma \in Y$ be fixed and let

$$
S=\{u \in H: \psi(u)=\gamma\} .
$$

Since $\psi$ is completely continuous, i.e., continuous from $H$ endowed with the weak topology to $Y$ with the norm topology, $S$ is weakly closed in $H$. We assume that $S \neq \varnothing$, that $j(u)<+\infty$, for some $u \in S$ and $\varphi$ is bounded from below on $S$ in the sense that there exist constants $c>0$ and $0 \leq \beta<2$ such that

$$
\varphi(u) \geq-c\|u\|^{\beta}
$$


We define

$$
F: H \rightarrow \mathbb{R}
$$

by

$$
F(u)=\varphi(u)+j(u) .
$$

Then $F$ is weakly lower semicontinuous on $S$.

We now consider the following minimization problem:

$$
F(u)=\min _{v \in S} F(v),
$$

i.e., the problem is to show the existence of $u \in S$ such that (2.3) holds.

\subsection{Functionals with property $(P)$}

Definition. We say that the functional $F$ (or the pair $(\varphi, j))$ has property $(P)$ on $S$ whenever the following hold:

If $\left\{v_{n}\right\} \subset S$ is any sequence in $S$ satisfying, as $n \rightarrow \infty$,

$$
\begin{aligned}
& \left\|v_{n}\right\| \rightarrow \infty, \\
& w_{n}=\frac{v_{n}}{\left\|v_{n}\right\|} \rightarrow 0, \\
& \limsup _{n \rightarrow \infty} \frac{\varphi\left(v_{n}\right)}{\left\|v_{n}\right\|^{2}} \leq 0,
\end{aligned}
$$

(where, as customary, $\rightarrow$ denotes weak convergence) then there exists $v_{0} \in S$ such that

$$
\limsup _{n \rightarrow \infty} F\left(v_{n}\right)>F\left(v_{0}\right)
$$

Our main abstract existence result for problem (2.3) will involve functionals having property $(P)$. Before stating and proving this result we shall provide some conditions guaranteeing that property $(P)$ holds.

Proposition 2.1. Let $K$ be a closed convex subset of $V, S \subset K, 0 \in K$, and assume that $A$ is nonnegative, i.e., $\langle A u, u\rangle \geq 0, u \in K$, and assume that: If $\left\{v_{n}\right\}$ is a sequence in $K$ such that

$$
\left\|v_{n}\right\| \rightarrow \infty, \quad \frac{v_{n}}{\left\|v_{n}\right\|} \rightarrow 0
$$

and

$$
\frac{\varphi\left(v_{n}\right)}{\left\|v_{n}\right\|^{2}} \rightarrow 0
$$

then

$$
\limsup _{n \rightarrow \infty} \frac{1}{\left\|v_{n}\right\|} F\left(v_{n}\right)>0 .
$$

Then $F$ has property $(P)$ on $S$.

Proof. Let $\left\{u_{n}\right\} \subset S$ be a sequence such that (2.4) holds, then since $\left\{u_{n}\right\} \subset K$ and (2.6) is valid we get by assumption that (2.7) is true. Since $\left\|v_{n}\right\| \rightarrow \infty$ we must have that

$$
\limsup _{n \rightarrow \infty} F\left(v_{n}\right)=\infty
$$


and therefore

$$
\limsup _{n \rightarrow \infty} F\left(v_{n}\right)>F(v)
$$

for all $v \in S$ with $j(v)<+\infty$; hence (2.5) holds.

The property described in Proposition 2.1 is the property called $P$-coerciveness used in [ASV3].

Proposition 2.2. Let $K$ be as above and assume that $F$ satisfies: If $\left\{v_{n}\right\}$ is a sequence in $S$ such that

$$
\begin{aligned}
& \left\|v_{n}\right\| \rightarrow \infty, \quad w_{n}=\frac{v_{n}}{\left\|v_{n}\right\|} \rightarrow w, \\
& \sup _{n \in \mathbb{N}} F\left(v_{n}\right)<+\infty
\end{aligned}
$$

then

$$
w_{n}=\frac{v_{n}}{\left\|v_{n}\right\|} \rightarrow w
$$

Then $F$ has property $(P)$.

Proof. Let $\left\{u_{n}\right\} \subset S$ be a sequence such that (2.4) holds, we claim that

$$
\limsup _{n \rightarrow \infty} F\left(u_{n}\right)=+\infty
$$

and hence complete the proof as in Proposition 2.1. If the claim were false we get that

$$
F\left(v_{n}\right) \leq c, \quad n \in \mathbb{N},
$$

for some constant $c$ and hence by assumption

$$
w_{n} \rightarrow 0 \text {, }
$$

a contradiction to $\left\|w_{n}\right\|=1$.

Proposition 2.3. Let $K$ be as above and assume again that $A$ is nonnegative and there exist $c>0, P_{0}, P_{1}: H \rightarrow \mathbb{R}^{+}$such that for all $v \in K$ satisfying

$$
\|v\|=1, \quad \lambda v \in K, \quad \lambda \geq 1
$$

one has

$$
\frac{1}{\lambda}\langle A(\lambda v), v\rangle+P_{0}(v)+P_{1}(v)+j^{+}(v) \geq c,
$$

$\left(j^{+}(v)=\max \{j(v), 0\}\right), P_{0}(K)$ is bounded and there exists $s>0$ such that

$$
P_{0}(\lambda u) \leq \lambda^{s} P_{0}(u), \quad \forall \lambda \in[0,1], \quad \forall u \in K
$$

Further

$$
P_{1}\left(v_{n}\right) \rightarrow 0, \quad \text { whenever } v_{n} \rightarrow 0,\left\{v_{n}\right\} \subset K .
$$

Then $F$ satisfies property $(P)$.

Proof. The above result is from [ASV3], where it is shown that the above conditions imply the hypotheses of Proposition 2.1 and hence property $(P)$ holds.

2.3 Existence results. Let the assumptions and notations of section 2.1 hold. We are now able to establish existence results for problems (2.3) for functionals 
that satisfy property $(P)$ provided certain compatibility conditions are satisfied. Before proceeding to the abstract results we need one further piece of information from [ASV1].

Associated with the functional $j$ we have the following functional $j_{\infty}: H \rightarrow$ $\mathbb{R} \cup\{\infty\}$ defined by $j$

$$
j_{\infty}(w)=\lim _{t \rightarrow \infty} \frac{1}{t} j(t w) .
$$

This functional has the following properties as stated and proved in [ASV1].

Lemma 2.4. The functional $j_{\infty}$ satisfies:

(1) $j_{\infty}$ is convex and lower semicontinuous with $j_{\infty}(0)=0$,

(2) $j(u+v) \leq j(u)+j_{\infty}(v), \forall u, v \in H$,

(3) $j_{\infty}(\lambda w)=\lambda j_{\infty}(w) \quad \forall \lambda \geq 0, \forall w \in H$,

(4) If $\frac{u_{n}}{\left\|u_{n}\right\|} \rightarrow w$ and $\left\|u_{n}\right\| \rightarrow \infty$, then

$$
j_{\infty}(w) \leq \liminf _{n \rightarrow \infty} \frac{j\left(u_{n}\right)}{\left\|u_{n}\right\|} .
$$

We are now in a position to state and prove our main existence result and establish several consequences.

Theorem 2.5. Let $F$ satisfy property $(P)$ on $S$ and suppose the following compatibility condition is satisfied: If $w \in H$ is such that there exists a sequence $\left\{u_{n}\right\} \subset H$ such that

$$
\begin{aligned}
& \left\|u_{n}\right\| \rightarrow \infty, \quad w_{n}=\frac{u_{n}}{\left\|u_{n}\right\|} \rightarrow w, \\
& \limsup _{n \rightarrow \infty} \frac{1}{\left\|u_{n}\right\|} \varphi\left(u_{n}\right)+j_{\infty}(w) \leq 0, \\
& \lim _{n \rightarrow \infty} \frac{\psi\left(u_{n}\right)}{\left\|u_{n}\right\|^{\lambda}}=0, \quad \forall \lambda>0,
\end{aligned}
$$

Then we have

$$
\left\{\begin{aligned}
u-w \in S, & \forall u \in S, \\
F(u-w) \leq F(u), & \forall u \in S .
\end{aligned}\right.
$$

Under the above assumptions the minimization problem (2.3) has a solution $u \in S$.

Proof. We employ the method of elliptic regularization (see, e.g., [KS]), i.e., we consider the perturbed functionals

$$
F_{\epsilon}(u)=F(u)+\epsilon\|u\|^{2}, \quad u \in H, \epsilon>0,
$$

and show that the problems

$$
F_{\epsilon}(u)=\min _{v \in S} F_{\epsilon}(v)
$$

have solutions $\left\{u_{\epsilon}\right\} \subset S$. We next show that the family $\left\{u_{\epsilon}\right\}$ is uniformly bounded, From which will follow that there exists a subsequence $\left\{u_{\epsilon_{n}}\right\}, \epsilon_{n} \downarrow 0$, and $u$ such that

$$
u_{\epsilon_{n}} \rightarrow u
$$


Since $S$ is weakly closed, $u \in S$. Further, since $F$ is weakly lower semicontinuous,

$$
\begin{aligned}
F(u) & \leq \liminf _{n \rightarrow \infty} F\left(u_{\epsilon_{n}}\right) \\
& \leq \liminf _{n \rightarrow \infty}\left(F\left(u_{\epsilon_{n}}\right)+\epsilon_{n}\left\|u_{\epsilon_{n}}\right\|^{2}\right) \\
& \leq \liminf _{n \rightarrow \infty}\left(F(v)+\epsilon_{n}\|v\|^{2}\right), \quad \forall v \in S \\
& =F(v), \quad \forall v \in S .
\end{aligned}
$$

Hence

$$
F(u)=\min _{v \in S} F(v) .
$$

Thus consider problem (2.16). Since $j$ is lower semicontinuous and convex, there exists $\ell \in H$ and $c \in \mathbb{R}$ such that

$$
j(u) \geq\langle\ell, u\rangle+c, \quad \forall u \in H .
$$

Hence

$$
\begin{aligned}
F_{\epsilon}(u) & =\epsilon\|u\|^{2}+\langle A u, u\rangle+j(u) \\
& \geq \epsilon\|u\|^{2}-c\|u\|^{\beta}-|\langle\ell, u\rangle|-|c|,
\end{aligned}
$$

since $0 \leq \beta<2$ we obtain immediately that

$$
F_{\epsilon}(u) \rightarrow \infty \quad \text { as } \quad\|u\| \rightarrow \infty,
$$

i.e., $F_{\epsilon}$ is coercive on $H$, hence on $S$. Since $S$ is weakly closed in $H$, there exists $u_{\epsilon} \in S$ such that

$$
F_{\epsilon}\left(u_{\epsilon}\right)=\min _{v \in S} F_{\epsilon}(v) .
$$

It suffices therefore to show that $\left\{u_{\epsilon}\right\}$ is bounded. Suppose this is not the case. Then there exists a subsequence

$$
\left\{u_{n}=u_{\epsilon_{n}}\right\} \subset\left\{u_{\epsilon}\right\}, \quad \epsilon_{n} \downarrow 0,
$$

such that

$$
\left\|u_{n}\right\| \rightarrow \infty, \quad n \rightarrow \infty .
$$

It follows from (2.17) that for all $v \in S$

$$
F\left(u_{n}\right)+\epsilon_{n}\left\|u_{n}\right\|^{2} \leq F(v)+\epsilon_{n}\|v\|^{2} .
$$

Hence

$$
\begin{aligned}
\limsup _{n \rightarrow \infty} \frac{1}{\left\|u_{n}\right\|} F\left(u_{n}\right) & \leq \limsup _{n \rightarrow \infty} \frac{1}{\left\|u_{n}\right\|}\left(F\left(u_{n}\right)+\epsilon_{n}\left\|u_{n}\right\|^{2}\right) \\
& \leq \limsup _{n \rightarrow \infty} \frac{1}{\left\|u_{n}\right\|}\left(F(v)+\epsilon_{n}\|v\|^{2}\right) \\
& =0 .
\end{aligned}
$$

Passing to a subsequence, if necessary, we may assume that

$$
w_{n}=\frac{u_{n}}{\left\|u_{n}\right\|} \rightarrow w
$$

Using the properties of the functional $j_{\infty}$ (see Lemma 2.4) we have

$$
j_{\infty}(w) \leq \liminf _{n \rightarrow \infty} \frac{j\left(u_{n}\right)}{\left\|u_{n}\right\|} .
$$


Hence

$$
\begin{aligned}
\limsup _{n \rightarrow \infty} \frac{1}{\left\|u_{n}\right\|} \varphi\left(u_{n}\right)+j_{\infty}(w) & \leq \limsup _{n \rightarrow \infty} \frac{1}{\left\|u_{n}\right\|} \varphi\left(u_{n}\right)+\liminf _{n \rightarrow \infty} \frac{j\left(u_{n}\right)}{\left\|u_{n}\right\|} \\
& \leq \limsup _{n \rightarrow \infty} \frac{1}{\left\|u_{n}\right\|} F\left(u_{n}\right) \\
& \leq 0 .
\end{aligned}
$$

Further, since $\left\{u_{n}\right\} \subset S, \psi\left(u_{n}\right)=\gamma$, we must have

$$
\limsup _{n \rightarrow \infty} \frac{\psi\left(u_{n}\right)}{\left\|u_{n}\right\|^{\lambda}}=0, \quad \lambda>0 .
$$

Thus (2.13) holds and consequently, by hypothesis, (2.14) must hold. In particular,

$$
u_{n}-w \in S, \quad F\left(u_{n}-w\right) \leq F\left(u_{n}\right), \quad \forall n .
$$

Letting $v=u_{n}-w$ in (2.18), we get

$$
\begin{aligned}
F\left(u_{n}\right)+\epsilon_{n}\left\|u_{n}\right\|^{2} & \leq F\left(u_{n}-w\right)+\epsilon_{n}\left\|u_{n}-w\right\|^{2} \\
& \leq F\left(u_{n}\right)+\epsilon_{n}\left\|u_{n}-w\right\|^{2} .
\end{aligned}
$$

Thus

$$
\left\|u_{n}\right\|^{2} \leq\left\|u_{n}-w\right\|^{2}, \quad \forall n
$$

or

$$
\left\|u_{n}\right\|^{2} \leq\left\|u_{n}\right\|^{2}-2\left\langle u_{n}, w\right\rangle+\|w\|^{2}
$$

and hence

$$
\left\langle u_{n}, w\right\rangle \leq \frac{1}{2}\|w\|^{2}
$$

Therefore

$$
\left\langle w_{n}, w\right\rangle=\frac{1}{\left\|u_{n}\right\|}\left\langle u_{n}, w\right\rangle \leq \frac{\|w\|^{2}}{2\left\|u_{n}\right\|},
$$

and since $w_{n} \rightarrow w$ we conclude $w=0$, i.e.

$$
w_{n} \rightarrow 0 .
$$

Using (2.18) again and dividing the inequality by $\left\|u_{n}\right\|^{2}$, we obtain

$$
\limsup _{n \rightarrow \infty} \frac{1}{\left\|u_{n}\right\|^{2}} F\left(u_{n}\right) \leq 0
$$

i.e.,

$$
\limsup _{n \rightarrow \infty}\left\{\frac{1}{\left\|u_{n}\right\|^{2}} \varphi\left(u_{n}\right)+\frac{1}{\left\|u_{n}\right\|^{2}} j\left(u_{n}\right)\right\} \leq 0 .
$$

Since $j$ is convex and $j(0)=0$, we have

$$
\begin{aligned}
\liminf _{n \rightarrow \infty} \frac{j\left(u_{n}\right)}{\left\|u_{n}\right\|^{2}} & \geq \liminf _{n \rightarrow \infty} j\left(\frac{u_{n}}{\left\|u_{n}\right\|^{2}}\right) \\
& \geq j\left(\lim _{n \rightarrow \infty} \frac{u_{n}}{\left\|u_{n}\right\|^{2}}\right)=0 .
\end{aligned}
$$

Hence (2.19) implies

$$
\limsup _{n \rightarrow \infty} \frac{1}{\left\|u_{n}\right\|^{2}} \varphi\left(u_{n}\right) \leq 0
$$


We thus have all three conditions in the definition of property $(P)$ holding. Consequently there must exist $u_{0} \in S$ such that

$$
\limsup _{n \rightarrow \infty} F\left(u_{n}\right)>F\left(u_{0}\right) \text {. }
$$

But (2.18) implies

$$
F\left(u_{n}\right) \leq F_{\epsilon_{n}}\left(u_{n}\right) \leq F_{\epsilon_{n}}\left(u_{0}\right)=F\left(u_{0}\right)+\epsilon_{n}\left\|u_{0}\right\|^{2},
$$

and we obtain a contradiction to (2.20).

\section{CRitical POINT THEOREMS}

In this section we shall use Theorem 2.5 together with Liusternik's theorem (Lagrange multipliers) (see [K]) to establish some existence results for critical points of certain functionals.

Let us now assume that

$$
a: H \times H \rightarrow \mathbb{R}
$$

is a continuous bilinear form which is positive, i.e.,

$$
a(u, u) \geq 0, \quad \forall u \in H .
$$

Then

$$
\varphi: H \rightarrow \mathbb{R}
$$

given by

$$
\varphi(u)=a(u, u)
$$

is a nonnegative weakly lower semicontinuous functional on $H$. Let us assume that

$$
\operatorname{dim} W=\operatorname{dim} \operatorname{ker} a=\operatorname{dim}\{u: a(u, u)=0\}<+\infty,
$$

and there exists $c>0$ such that

$$
\varphi(u) \geq c\|u\|^{2}, \quad \forall u \in W^{\perp},
$$

i.e., $\varphi$ is coercive on $W^{\perp}$.

Remark 3.1. Throughout we shall denote by ker of a mapping, say $\varphi, \operatorname{ker} \varphi=$ $\varphi^{-1}\{0\}$; this notation is not intended to convey that $\operatorname{ker} \varphi$ is a linear space, even though in many situations, it will be; see, e.g., (3.3) above.

Let

$$
\psi: H \rightarrow \mathbb{R}
$$

be a weakly continuous functional homogeneous of degree $\alpha>1, \alpha \neq 2$, i.e.,

$$
\psi(\lambda u)=\lambda^{\alpha} \psi(u), \quad \forall \lambda \geq 0, u \in H,
$$

and let $S$ be given by

$$
S=\{u \in H: \psi(u)=\gamma\},
$$

which we assume to be nonempty.

We have the following consequence of Theorem 2.5. 
Corollary 3.2. Let $\varphi$ and $\psi$ satisfy the above conditions and assume

$$
\psi(u) \neq 0, \quad \forall u \in(W=\operatorname{ker} a) \backslash\{0\} .
$$

Then there exists $u \in S$ such that

$$
\varphi(u)=\min _{v \in S} \varphi(v) .
$$

Proof. We check the conditions of Theorem 2.5. To this end we let

$$
\langle A u, v\rangle=a(u, v)
$$

and $j \equiv 0$. We shall prove that $F=\varphi$ satisfies property $(P)$. We shall show this by invoking Proposition 2.3, choosing $P_{0}=0$ and $P_{1}(u)=\|\Pi u\|^{2}$, where $\Pi: H \rightarrow W$ is the orthogonal projection of $H$ onto $W=\operatorname{ker} \varphi$. Since $\operatorname{dim} W<+\infty, \Pi$ is a compact mapping proving that $P_{1}\left(u_{n}\right) \rightarrow 0$, whenever $u_{n} \rightarrow 0$. Hence (2.11) and (2.12) hold. Now for $u \in H,\|u\|=1, \lambda \geq 1$

$$
\begin{aligned}
& \frac{1}{\lambda}\langle A(\lambda u), u\rangle+P_{1}(u)=a(u, u)+P_{1}(u) \\
& \quad=a(u-\Pi(u), u-\Pi(u))+\|\Pi(u)\|^{2} \quad\left(u-\Pi(u) \in W^{\perp}\right) \\
& \quad \geq c\|u-\Pi(u)\|^{2}+\|\Pi(u)\|^{2} \\
& \quad \geq \min (c, 1)\left(\|u-\Pi(u)\|^{2}+\|\Pi(u)\|^{2}\right) \\
& \quad \geq \min (c, 1)>0 .
\end{aligned}
$$

Thus $(2.10)$ is true, showing that property $(P)$ holds.

We next check the compatibility condition of Theorem 2.5. To this end, let $\left\{u_{n}\right\},\left\{w_{n}\right\}$, and $w$ satisfy (2.13). We shall show that $w=0$ and hence (2.14) will obviously hold. We have (since $j_{\infty} \equiv 0$ )

$$
\limsup _{n \rightarrow \infty} \frac{1}{\left\|u_{n}\right\|} a\left(u_{n}, u_{n}\right) \leq 0
$$

(see (2.13)). Hence

$$
\limsup _{n \rightarrow \infty} a\left(w_{n}, w_{n}\right)=\limsup _{n \rightarrow \infty} \frac{1}{\left\|u_{n}\right\|^{2}} a\left(u_{n}, u_{n}\right) \leq 0 .
$$

Since $\varphi$ is weakly lower semicontinuous

$$
\varphi(w) \leq \liminf _{n \rightarrow \infty} \varphi\left(w_{n}\right) \leq 0,
$$

and therefore

$$
\varphi(w)=a(w, w)=0,
$$

i.e., $w \in W=\operatorname{ker} a$. Now using (2.13) for $\psi$ and the weak continuity of $\psi$, we get

$$
0=\lim _{n \rightarrow \infty} \frac{1}{\left\|u_{n}\right\|^{\alpha}} \psi\left(u_{n}\right)=\lim _{n \rightarrow \infty} \psi\left(\frac{u_{n}}{\left\|u_{n}\right\|}\right)=\lim _{n \rightarrow \infty} \psi\left(w_{n}\right)=\psi(w),
$$

which by hypothesis implies that $w=0$.

Corollary 3.3. Assume the conditions of Corollary 3.2 and that $\psi$ also satisfies:

$$
\left\{\begin{array}{l}
\exists u \in H \quad \text { such that } \psi(u)<0, \\
\psi(u)>0, \quad \forall u \in W \backslash\{0\} .
\end{array}\right.
$$


Then the functional $f$ defined by

$$
f(u)=\varphi(u)+\psi(u)
$$

has a nontrivial critical point.

Proof. If $u \in H$ is such that $\psi(u)=M<0$, then $\psi\left(|M|^{-1 / \alpha} u\right)=-1$. Thus

$$
S=\{u: \psi(u)=-1\} \neq \varnothing .
$$

Since $\psi$ is homogeneous, we have that for $u \in S$

$$
\left\langle\psi^{\prime}(u), u\right\rangle=\alpha \psi(u)=-\alpha \neq 0 .
$$

Hence $\psi^{\prime}(u) \neq 0, u \in S$. Let $u$ be the minimizer of Corollary 3.2. Then we obtain from Liusternik's theorem (see $[\mathrm{K}]$ ) the existence of a Lagrange multiplier $\mu \in \mathbb{R}$ such that

$$
\varphi^{\prime}(u)+\mu \psi^{\prime}(u)=0
$$

Hence

$$
0=\left\langle\varphi^{\prime}(u), u\right\rangle+\mu\left\langle\psi^{\prime}(u), u\right\rangle=2 \varphi(u)+\mu \alpha \psi(u)=2 \varphi(u)-\mu \alpha .
$$

Since $\varphi(u)>0$ for $u \in S$, we get $\alpha>0$. Rescaling, i.e., putting $v=\mu^{\frac{1}{\alpha-2}} u$, we see that $v$ is a critical point of $f$.

Remark 3.4. Corollary 3.2 is the major result from [BTW].

The following corollary contains Corollary 3.2 and Corollary 3.3 as special cases.

Corollary 3.5. Let $\varphi$ and $\psi$ be as above (i.e., satisfy the conditions (3.1)-(3.6)) and assume that

$$
\psi(v-u)=\psi(v), \quad \forall v \in H, \quad \forall u \in W \cap \operatorname{ker} \psi,
$$

where $\operatorname{ker} \psi=\{u: \psi(u)=0\}$. Then the problem (3.8) has a solution.

Proof. We have shown above already that property $(P)$ holds. We hence must verify that (2.13) and (2.14) are true. Thus let $w,\left\{w_{n}\right\}$, and $\left\{u_{n}\right\}$ be as in these conditions. We know that $\varphi(w)=\psi(w)=0$ (see Corollary 3.2), i.e., $w \in \operatorname{ker} \varphi \cap \operatorname{ker} \psi$. By hypothesis we have (3.12) holding, i.e., $\psi(v-w)=\psi(v)$, $\forall v \in H$. Hence $v-w \in S$, whenever $v \in S$. Moreover, since $w \in \operatorname{ker} \varphi=W$, we have

$$
a(w, v)+a(v, w)=\left\langle\varphi^{\prime}(w), v\right\rangle=0, \forall v \in W
$$

Hence

$$
\varphi(v-w)=\varphi(v)+\varphi(w)-[a(v, w)+a(w, v)]=\varphi(v)
$$

proving (2.14) and hence the result follows.

Corollary 3.6. Suppose again that $\psi \in C^{1}$ and that $\varphi$ and $\psi$ satisfy the conditions at the beginning of this section and that

(i) $\psi(u)<0$, for some $u \in H$,

(ii) $\psi(u) \geq 0, \quad \forall u \in W$ and 
(iii) if $u \in W$ is such that $\psi(u)=0$, then $\psi(v-u)=\psi(v), \forall v \in H$.

Then the functional $f$ given by (3.10) has a critical point.

Proof. It follows from (i) that

$$
S=\{u \in H: \psi(u)=-1\} \neq \varnothing .
$$

Since $\left\langle\psi^{\prime}(u), u\right\rangle=-\alpha$, we have $\psi^{\prime}(u) \neq 0, \forall u \in S$, and we again may apply Liusternik's theorem ([K]) together with Corollary 3.5 to obtain

$$
\varphi^{\prime}(u)+\mu \psi^{\prime}(u)=0
$$

for some $\mu \in \mathbb{R}$.

As in Corollary 3.3 we have

$$
2 \varphi(u)-\mu \alpha=0
$$

and since $\psi \geq 0$ on $\operatorname{ker} \varphi$ we have

$$
S \cap \operatorname{ker} \varphi=\varnothing .
$$

This implies that $\varphi>0$ on $S$. Hence $\varphi(u)>0$ and $\mu>0$. Again the rescaling

$$
v=\lambda^{-1} u=\mu^{\frac{1}{\alpha-2}} u
$$

yields the desired conclusion.

\section{EXtensions to RefleXive Banach SPACES}

4.1 Assumptions and notations. Let $V$ be a real reflexive Banach space with norm $\|\cdot\|$, dual $V^{*}$ and the pairing between $V$ and $V^{*}$ given by $\langle\cdot, \cdot\rangle$. Let

$$
A: V \rightarrow V^{*}
$$

be a mapping such that the functional

$$
\varphi: V \rightarrow \mathbb{R}
$$

given by

$$
u \mapsto\langle A u, u\rangle
$$

is weakly lower semicontinuous.

Concerning $Y, \psi, S, \varphi, j$, and $F=\varphi+j$ we make the same assumptions as in section 2.1 (replacing $H$ by $V$ everywhere) and we consider the minimization problem

$$
F(u)=\min _{v \in S} F(v) .
$$

As before, we introduce a property which together with certain compatibility conditions on $S$ will imply the boundedness of the solution set of the regularized problems. As we shall see this property will be an extension of the property $(P)$ introduced earlier. We hence shall employ the same terminology. 


\subsection{Functionals with property $(P)$.}

Definition. We say that the functional $F$ (or the pair $(\varphi, j)$ or $\varphi$, if $j=0$ ) has property $(P)$ on $S$ whenever the following hold: There exists a constant $p>1$ such that: If $\left\{v_{n}\right\} \subset S$ is any sequence in $S$ satisfying, as $n \rightarrow \infty$,

$$
\begin{cases}\text { (a) } & \left\|v_{n}\right\| \rightarrow \infty, \\ \text { (b) } & w_{n}=\frac{v_{n}}{\left\|v_{n}\right\|}-w, \\ \text { (c) } & \left\|v_{n}\right\| \leq\left\|v_{n}-\lambda w\right\|, \quad \forall n, \quad \forall \lambda \geq 1, \\ \text { (d) } & \limsup _{n \rightarrow \infty} \frac{\varphi\left(v_{n}\right)}{\left\|v_{n}\right\|^{p}} \leq 0,\end{cases}
$$

then there exists $v_{0} \in S$ such that

$$
\limsup _{n \rightarrow \infty} F\left(v_{n}\right)>F\left(v_{0}\right) .
$$

We note that, in some sense, condition $(4.2(\mathrm{c}))$ is an orthogonality condition between $v_{n}$ and $w$ (see, e.g., $[\mathrm{J}]$ ).

The main abstract existence result for problem (4.1), as before, will involve functionals having property $(P)$. Again, before stating and proving this result we shall provide some conditions guaranteeing that property $(P)$ holds.

Proposition 4.1. Suppose that $F$ satisfies the conditions of the definition with (c) replaced by

$\left(\mathbf{c}^{\prime}\right) 1 \leq\left\|w_{n}-w\right\|, \forall n$ sufficiently large,

or

$\left(\mathrm{c}^{\prime \prime}\right)\|w\|<1$, provided $V$ is locally uniformly convex with respect to $\|\cdot\|$, or

$\left(\mathrm{c}^{\prime \prime \prime}\right) V$ is a Hilbert space, and $w=0$.

Then $F$ satisfies property $(P)$.

Proof. Suppose $\left\{v_{n}\right\}$ and $F$ are as above. We label the cases to be considered as $\left(\mathrm{c}^{\prime}\right),\left(\mathrm{c}^{\prime \prime}\right),\left(\mathrm{c}^{\prime \prime \prime}\right)$.

Case $\left(\mathrm{c}^{\prime}\right)$ : Since (by (a)) $\left\|v_{n}\right\| \geq 1$, for $n$ sufficiently large, we have from (c) that

$$
\left\|v_{n}\right\| \leq\left\|v_{n}-\right\| v_{n}\|w\|, \quad \forall n \text { sufficiently large, }
$$

hence

$$
1 \leq\left\|w_{n}-w\right\|, \quad \forall n \text { sufficiently large, }
$$

and property $(P)$ holds.

Case $\left(\mathrm{c}^{\prime \prime}\right)$ : We first note that if $V$ is reflexive then we may choose (by the Lindenstrauss-Asplund-Trojanski theorem) ([PS] or [T]) an equivalent norm on $V$ such that $V$ is locally uniformly convex with respect to the new norm. The local uniform convexity of $V$ yields the property that (see, e.g., [B])

$$
u_{n} \rightarrow u \text {, whenever } u_{n} \rightarrow u \text { and }\left\|u_{n}\right\| \rightarrow\|u\| \text {. }
$$

Suppose then that the conditions of the definition hold for a sequence $\left\{v_{n}\right\}$ but that $\left(\mathrm{c}^{\prime \prime}\right)$ is false, i.e., $\|w\| \geq 1$. We then have 


$$
\limsup _{n \rightarrow \infty}\left\|w_{n}\right\|=1 \leq\|w\| \text {. }
$$

However, since $\|\cdot\|$ is lower semicontinuous, it follows that

$$
\|w\| \leq \liminf _{n \rightarrow \infty}\left\|w_{n}\right\|
$$

and hence

$$
\|w\|=\lim _{n \rightarrow \infty}\left\|w_{n}\right\|
$$

and thus by the above property

$$
w_{n} \rightarrow w,
$$

contradicting the fact that for $n$ large

$$
1 \leq\left\|w_{n}-w\right\|
$$

which follows from (c).

Case $\left(\mathrm{c}^{\prime \prime \prime}\right)$ : Suppose $\left\{v_{n}\right\}$ is a sequence as in the definition. Choose $\lambda=1$ in (c) and get

$$
\left\|v_{n}\right\|^{2} \leq\left\|v_{n}-w\right\|^{2}=\left\|v_{n}\right\|^{2}+\|w\|^{2}-2\left\langle v_{n}, w\right\rangle
$$

$(\langle\cdot, \cdot\rangle$ is the inner product of $V$ now) and thus

implying that

$$
\left\langle w_{n}, w\right\rangle \leq \frac{\|w\|^{2}}{2\left\|v_{n}\right\|^{2}},
$$

$$
\|w\|^{2}=\lim _{n \rightarrow \infty}\left\langle w_{n}, w\right\rangle=0
$$

Hence $w=0$.

Thus in either of the three cases we have shown that property $(P)$ holds.

Proposition 4.2. Assume that $F$ satisfies: If $\left\{v_{n}\right\}$ is a sequence in $S$ such that

$$
\begin{aligned}
& \left\|v_{n}\right\| \rightarrow \infty, \quad w_{n}=\frac{v_{n}}{\left\|v_{n}\right\|} \rightarrow w, \\
& \sup _{n \in \mathbb{N}} F\left(v_{n}\right)<+\infty .
\end{aligned}
$$

Then

Then $F$ has property $(P)$.

$$
w_{n}=\frac{v_{n}}{\left\|v_{n}\right\|} \rightarrow w
$$

Proof. The proof is similar to the proof of Proposition 2.2, so we omit it.

The following example shows that the conditions given by Proposition 4.2 are a strict special case of property $(P)$, even in the case of Hilbert spaces.

Example. Let $H$ be an infinite dimensional Hilbert space and let

$$
F(u)=\frac{\|u\|}{1+\|u\|} .
$$

We shall show that $F$ is weakly lower semicontinuous, has property $(P)$, yet does not satisfy the conditions of Proposition 4.2. In fact, suppose that $\left\{u_{n}\right\}$ is a sequence with $u_{n} \rightarrow u$. Select a subsequence $\left\{u_{n_{k}}\right\} \subset\left\{u_{n}\right\}$ such that

$$
\lim _{k \rightarrow \infty} F\left(u_{n_{k}}\right)=\liminf _{n \rightarrow \infty} F\left(u_{n}\right)
$$


Since $\left\{u_{n_{k}}\right\}$ is bounded, we may assume, by passing to a subsequence, if necessary, that $\left\{\left\|u_{n_{k}}\right\|\right\}$ is convergent. Moreover, by the weak lower semicontinuity of the norm, we have

$$
\|u\| \leq \lim _{k \rightarrow \infty}\left\|u_{n_{k}}\right\|
$$

Since the mapping

$$
G: \mathbb{R}^{+} \rightarrow \mathbb{R}^{+}, \quad x \mapsto \frac{x}{1+x}
$$

is continuous and increasing, we get

$$
\begin{aligned}
F(u) & =G\left(\left\|u_{i}\right\|\right) \leq G\left(\lim _{k \rightarrow \infty}\left\|u_{n_{k}}\right\|\right)=\lim _{k \rightarrow \infty} G\left(\left\|u_{n_{k}}\right\|\right) \\
& =\lim _{k \rightarrow \infty} F\left(u_{n_{k}}\right)=\liminf _{n \rightarrow \infty} F\left(u_{n}\right) .
\end{aligned}
$$

Hence $F$ is weakly lower semicontinuous. To show that $F$ has property $(P)$ we use the definition. Let $\left\{v_{n}\right\}$ be such a sequence, then, since $\left\|v_{n}\right\| \rightarrow \infty$, we have that

$$
\limsup _{n \rightarrow \infty} F\left(v_{n}\right)=1>F(0),
$$

i.e., (4.3) holds and hence $F$ has property $(P)$. Since $H$ is infinite dimensional, we may choose a sequence $\left\{u_{n}\right\} \subset H,\left\|u_{n}\right\|=1$ such that it contains no convergent subsequences. Let $v_{n}=n u_{n}$, then $\left\|v_{n}\right\| \rightarrow \infty$. By passing to a subsequence, we may assume that

$$
u_{n}=\frac{v_{n}}{\left\|v_{n}\right\|}-w .
$$

Also, since $F$ is bounded all the conditions of Proposition 4.2 hold, yet $u_{n}=$ $\frac{v_{n}}{\left\|v_{n}\right\|}$ does not converge to $w$.

We shall give a useful consequence of Proposition 4.2 which yields property $(P)$ for functionals $F$ which have the property that the associated $\varphi$ is coercive off its zero set which is assumed a finite dimensional linear space. We prove the following.

Proposition 4.3. Suppose that $\varphi$ is nonnegative and positive homogeneous of degree $p>1$. Further assume that

$$
\operatorname{ker} \varphi=\{u: \varphi(u)=0\}
$$

is a finite dimensional subspace of $V$ such that

$$
\varphi(u+v)=\varphi(v), \forall v \in V, \forall u \in \operatorname{ker} \varphi .
$$

Furthermore assume that $V=\operatorname{ker} \varphi \oplus X$, where $X$ is a closed subspace of $V$ and $\left.\varphi\right|_{X}$ is coercive in the sense that there exists $c>0$ such that

$$
\varphi(v) \geq c\|v\|^{p}, \forall v \in X .
$$

Then $F=\varphi+j$ satisfies property $(P)$ for any $j$ satisfying our stated assumptions.

Proof. We shall show that the conditions of Proposition 4.2 are met. To this end let $\left\{v_{n}\right\}$ be a sequence satisfying

$$
\left\|v_{n}\right\| \rightarrow \infty, \quad \frac{v_{n}}{\left\|v_{n}\right\|} \rightarrow w
$$


and

$$
\sup F\left(v_{n}\right)<+\infty
$$

Since $j$ is convex and lower semicontinuous, there exist constants $a, b \in \mathbb{R}$ such that

$$
j(u) \geq a\|u\|+b, \quad \forall u \in V .
$$

Hence

$$
\begin{aligned}
0 & \geq \limsup _{n \rightarrow \infty} \frac{1}{\left\|v_{n}\right\|^{p}}\left[\varphi\left(v_{n}\right)+j\left(v_{n}\right)\right] \\
& \geq \limsup _{n \rightarrow \infty} \frac{\varphi\left(v_{n}\right)}{\left\|v_{n}\right\|^{p}}+\liminf _{n \rightarrow \infty} \frac{a\left\|v_{n}\right\|+b}{\left\|v_{n}\right\|^{p}} \\
& =\limsup _{n \rightarrow \infty} \varphi\left(\frac{v_{n}}{\left\|v_{n}\right\|}\right) \\
& =\limsup _{n \rightarrow \infty} \varphi\left(w_{n}\right) \\
& \geq \liminf _{n \rightarrow \infty} \varphi\left(w_{n}\right) \\
& \geq \varphi(w) \geq 0 .
\end{aligned}
$$

Hence

$$
w \in \operatorname{ker} \varphi \text { and } \lim _{n \rightarrow \infty} \varphi\left(w_{n}\right)=0 .
$$

It follows from (4.4) that $v_{n}=\xi_{n}+\eta_{n}, \xi_{n} \in \operatorname{ker} \varphi, \eta_{n} \in X$. Hence (4.3) and (4.5) imply

$$
\varphi\left(v_{n}\right)=\varphi\left(\eta_{n}\right) \geq c\left\|\eta_{n}\right\|^{p}
$$

and

$$
\varphi\left(w_{n}\right)=\frac{\varphi\left(v_{n}\right)}{\left\|v_{n}\right\|^{p}} \geq c\left(\frac{\left\|\eta_{n}\right\|}{\left\|v_{n}\right\|}\right)^{p}=c\left\|\frac{\eta_{n}}{\left\|v_{n}\right\|}\right\|^{p}
$$

and we conclude by (4.7) that

$$
\frac{\eta_{n}}{\left\|v_{n}\right\|} \rightarrow 0
$$

Since $w_{n} \rightarrow 0$, it follows from (4.6) that $\frac{\xi_{n}}{\left\|v_{n}\right\|} \rightarrow 0$. Since $\operatorname{ker} \varphi$ is finite dimensional, we conclude that $\frac{\xi_{n}}{\left\|v_{n}\right\|} \rightarrow 0$. We hence conclude that $F$ has property $(P)$.

4.3 Existence results. Let us consider the analogue of Theorem 2.5 for the Banach space case. We have:

Theorem 4.4. Let $F$ satisfy property $(P)$ on $S$ and suppose the following compatibility condition is satisfied: If $w \in V$ is such that there exists a sequence $\left\{u_{n}\right\} \subset V$ such that

$$
\begin{aligned}
& \left\|u_{n}\right\| \rightarrow \infty, \quad w_{n}=\frac{u_{n}}{\left\|u_{n}\right\|} \rightarrow w, \\
& \limsup _{n \rightarrow \infty} \frac{1}{\left\|u_{n}\right\|} \varphi\left(u_{n}\right)+j_{\infty}(w) \leq 0, \\
& \lim _{n \rightarrow \infty} \frac{\psi\left(u_{n}\right)}{\left\|u_{n}\right\|^{\lambda}}=0, \quad \forall \lambda>0 .
\end{aligned}
$$


Then we have

$$
\begin{cases}u-w \in S, & \forall u \in S, \\ F(u-w) \leq F(u), & \forall u \in S .\end{cases}
$$

Under the above assumptions the minimization problem (4.1) has a solution $u \in S$.

Proof. We follow the steps used to prove Theorem 2.5 and use again the method of elliptic regularization, i.e., we consider the perturbed functionals

$$
F_{\epsilon}(u)=F(u)+\epsilon\|u\|^{2}, \quad u \in V, \epsilon>0,
$$

and show that the problems

$$
F_{\epsilon}(u)=\min _{v \in S} F_{\epsilon}(v)
$$

have a uniformly bounded set of solutions $\left\{u_{\epsilon}\right\} \subset S$.

We may proceed, as in the proof of Theorem 2.5 and reach the point that

$$
\left\|u_{n}\right\|^{2} \leq\left\|u_{n}-w\right\|^{2}, \quad \forall n .
$$

From this it follows that for $\lambda \geq 1$, or $t=\frac{1}{\lambda} \in(0,1]$ that

$$
\left\|u_{n}\right\| \leq\left\|u_{n}-w\right\| \leq(1-t)\left\|u_{n}\right\|+t\left\|u_{n}-\lambda w\right\|
$$

or

$$
\left\|u_{n}\right\| \leq\left\|u_{n}-\lambda w\right\|, \quad \forall n, \quad \forall \lambda \geq 1
$$

We hence have condition $(4.2(\mathrm{c}))$ of property $(P)$ verified. We proceed further as in the proof of Theorem 2.5 by dividing by $\left\|u_{n}\right\|^{p}$ rather than $\left\|u_{n}\right\|^{2}$ and verify the other parts of condition (4.2). We now invoke property $(P)$ to complete the proof via a contradiction.

\section{A CRITICAL POINT THEOREM}

In this section we shall use Theorem 4.4 together with Liusternik's theorem (Lagrange multipliers) (see [K]) to establish an existence result for critical points of certain functionals. This result is an extension to reflexive Banach spaces of Corollaries 3.2, 3.3, 3.5, and 3.6.

Let us assume that

$$
\varphi: V \rightarrow \mathbb{R}
$$

is a nonnegative weakly lower semicontinuous functional on $V$, which is positive homogeneous of degree $p>1$ (see section 4.3). Concerning $\psi$ we assume that

$$
\psi: V \rightarrow \mathbb{R}
$$

is weakly continuous and positive homogeneous of degree $\alpha>1$.

We have the following consequence of Theorem 4.4.

Corollary 5.1. (a) Let $\varphi$ and $\psi$ satisfy the above conditions and assume that $\varphi$ has property $(P)$ on $S$. Suppose furthermore that

$$
\left\{\begin{array}{l}
\varphi(v-u) \leq \varphi(v), \\
\psi(v-u)=\psi(v), \quad \forall v \in V, \quad \forall u \in \operatorname{ker} \varphi \cap \operatorname{ker} \psi .
\end{array}\right.
$$


Then the minimization problem

$$
\varphi(u)=\min _{v \in S} \varphi(v)
$$

has a solution $u \in S$.

(b) If $\varphi, \psi$ belong to class $C^{1}$, and $\alpha \neq p$ and if

(i) $\psi(u)<0$, for some $u \in V$,

(ii) $\psi(u) \geq 0, \quad \forall u \in \operatorname{ker} \varphi$ and

(iii) if $u \in \operatorname{ker} \varphi$ is such that $\psi(u)=0$, then (5.1) holds.

Then the functional

$$
f=\varphi+\psi
$$

has a critical point.

Proof. We verify the conditions of Theorem 4.4 for the functional $F=\varphi$, i.e., $j=0$. Let $\left\{u_{n}\right\},\left\{w_{n}\right\}, w$ be as in (4.8). It follows immediately that

$$
\limsup _{n \rightarrow \infty} \frac{\varphi\left(u_{n}\right)}{\left\|u_{n}\right\|} \leq 0
$$

and hence, since $\left\|u_{n}\right\| \rightarrow \infty$

$$
\limsup _{n \rightarrow \infty} \frac{\varphi\left(u_{n}\right)}{\left\|u_{n}\right\|^{p}} \leq 0
$$

However,

and thus

$$
\varphi\left(w_{n}\right)=\varphi\left(\frac{u_{n}}{\left\|u_{n}\right\|}\right)=\frac{\varphi\left(u_{n}\right)}{\left\|u_{n}\right\|^{p}}
$$

$$
\limsup _{n \rightarrow \infty} \varphi\left(w_{n}\right) \leq 0
$$

Since $\varphi$ is weakly lower semicontinuous

$$
0 \leq \varphi(w) \leq \liminf _{n \rightarrow \infty} \varphi\left(w_{n}\right) \leq \limsup _{n \rightarrow \infty} \varphi\left(w_{n}\right) \leq 0,
$$

i.e., $\varphi(w)=0$ and $w \in \operatorname{ker} \varphi$. Using the complete continuity and $\alpha$-homogeneity of $\psi$ we deduce that $\psi(w)=0$. It follows therefore from (5.1) that $v-w \in S$, whenever $v \in S$ and $\varphi(v-w) \leq \varphi(v), \forall v \in V$. Hence (4.9) is true and we may apply Theorem 4.4.

(b) It follows from (i) that

$$
S=\{u: \psi(u)=-1\} \neq \varnothing .
$$

Also (ii) implies that the assumptions of (a) hold. Hence if $u$ is a solution of the minimization problem $(5.2)$, then $\psi^{\prime}(u) \neq 0$, since

$$
\left\langle\psi^{\prime}(u), u\right\rangle=\alpha \psi(u)=-\alpha \neq 0 .
$$

We may apply Liusternik's theorem $([\mathrm{K}])$ to obtain

$$
\varphi^{\prime}(u)+\mu \psi^{\prime}(u)=0
$$

for some $\mu \in \mathbb{R}$.

Then

$$
\begin{aligned}
0 & =\left\langle\varphi^{\prime}(u), u\right\rangle+\mu\left\langle\psi^{\prime}(u), u\right\rangle \\
& =p \varphi(u)+\mu \alpha \psi(u) \\
& =p \varphi(u)-\mu \alpha .
\end{aligned}
$$


Since $\psi \geq 0$ on $\operatorname{ker} \varphi$ we have

$$
S \cap \operatorname{ker} \varphi=\varnothing .
$$

This implies that $\varphi(u)>0, \mu>0$. Again the rescaling

$$
v=\mu^{\frac{1}{x-p}} u
$$

yields the desired conclusion

$$
\varphi^{\prime}(v)+\psi^{\prime}(v)=0 .
$$

\section{Applications}

In this section we shall consider some applications of the results derived above.

6.1 A problem with exponential nonlinearity. Let $\Omega \subset \mathbb{R}^{2}$ be a bounded domain with smooth boundary and consider the partial differential equation

$$
\Delta u+k e^{u}=h, \quad \text { in } \Omega
$$

subject to the (nonlocal) boundary conditions

$$
\left\{\begin{array}{l}
u \equiv \text { constant, on } \partial \Omega \\
\int_{\partial \Omega} \frac{\partial u}{\partial n}=0,
\end{array}\right.
$$

where $k$ and $h$ are given functions and $\frac{\partial u}{\partial n}$ is the normal derivative on $\partial \Omega$.

In this case we choose

$$
H=\left\{u \in H^{1}(\Omega):\left.u\right|_{\partial \Omega}=\text { constant }\right\},
$$

then $H$ is a (Hilbert) subspace of $H^{1}(\Omega)$ with respect to the $H^{1}$ norm

$$
\|u\|=\left(\int_{\Omega}\left[u^{2}+|\nabla u|^{2}\right]\right)^{1 / 2} .
$$

We assume that $k \in C(\bar{\Omega})$ and $h \in L^{2}(\Omega)$ and define

$$
\varphi(u)=\frac{1}{2}\langle A u, u\rangle, \quad u \in H,
$$

with

$$
\langle A u, v\rangle=\int_{\Omega} \nabla u \cdot \nabla v, \quad u, v \in H,
$$

and

$$
j(u)=\int_{\Omega} h u, \quad u \in H,
$$

then $j$ is a bounded linear functional, and $\operatorname{ker} \varphi=\mathbb{R}$.

We let

$$
S=\left\{u \in H: \psi(u)=\int_{\Omega} k e^{u}=0\right\},
$$

then $S \neq \varnothing$, whenever $k$ assumes both positive and negative values. Since $n=2$, it follows that

$$
\psi: H \rightarrow \mathbb{R}
$$


is a completely continuous mapping. This is an immediate consequence of Trudinger's theorem ([GT]) about the embedding of $H^{1}(\Omega)$ into the Orlicz space $L_{\varphi}(\Omega)$ with $\varphi(t)=e^{t^{2}}-1, t \geq 0$ (see also [A]), and compact embedding results between Orlicz spaces ([A]). Hence $S$ is weakly closed in $H$. Further $\psi \in C^{1}(H, \mathbb{R})$ and

$$
\left\langle\psi^{\prime}(u), v\right\rangle=\int_{\Omega} k e^{u} v, \quad u, v \in H .
$$

We have the following corollary.

Corollary 6.1. Assume that $k$ changes sign on $\Omega$ and that $\int_{\Omega} h=0$. Then there exists $u \in S$ such that

$$
\varphi(u)+j(u)=\min _{v \in S}[\varphi(v)+j(v)],
$$

where $\varphi$ and $j$ are given by (6.3) and (6.4), respectively.

Proof. We note that $F=\varphi+j$ satisfies property $(P)$ by an application of Proposition 2.3. We choose $P_{0} \equiv 0$ and $P_{1}(u)=\int_{\Omega} u^{2}$. Then we have for $u \in H,\|u\|=1$ and $\lambda \geq 1$

$$
\frac{1}{\lambda}\langle A(\lambda u), u\rangle+P_{1}(u)+j^{+}(u) \geq \int_{\Omega}|\nabla u|^{2}+\int_{\Omega} u^{2}=\|u\|^{2}=1 .
$$

Hence (2.10) holds and therefore property $(P)$ is valid.

Let now $\left\{w_{n}\right\},\left\{u_{n}\right\}$, and $w$ be as in (2.13) (we shall employ Theorem 2.5). We then have (since $j_{\infty}=j$ )

$$
\limsup _{n \rightarrow \infty} \frac{1}{\left\|u_{n}\right\|} \varphi\left(u_{n}\right)+j(w) \leq 0
$$

and therefore

$$
\limsup _{n \rightarrow \infty}\left[\frac{1}{\left\|u_{n}\right\|^{2}} \varphi\left(u_{n}\right)+\frac{j(w)}{\left\|u_{n}\right\|}\right] \leq 0,
$$

i.e.,

$$
\limsup _{n \rightarrow \infty} \varphi\left(w_{n}\right)=\limsup _{n \rightarrow \infty}\left\langle A w_{n}, w_{n}\right\rangle \leq 0 .
$$

Hence (by the weak lower semicontinuity) $\langle A w, w\rangle \leq 0$, and therefore $\langle A w$, $w\rangle=0$, i.e., $w \in \operatorname{ker} \varphi=\mathbb{R}$.

Let $u \in S$, we then have

$$
\psi(u-w)=\int_{\Omega} k e^{u-w}=e^{-w} \int_{\Omega} k e^{u}=0 .
$$

Hence $u-w \in S$ and moreover,

$$
\begin{aligned}
F(u-w) & =\langle A(u-w), u-w\rangle+j(u-w) \\
& =\langle A u, u\rangle+\int_{\Omega} h(u-w) \\
& =\langle A u, u\rangle+\int_{\Omega} h u=F(u),
\end{aligned}
$$

since $\int_{\Omega} h=0$. We may therefore employ Theorem 2.5 to obtain the conclusion.

It follows from the Fredholm alternative that there exists $u_{0} \in H$ such that

$$
\int_{\Omega} \nabla u_{0} \cdot \nabla v=\int_{\Omega} h v, \quad \forall v \in H,
$$

whenever $\int_{\Omega} h=0$. Using this fact, we obtain the following corollary. 
Corollary 6.2. Assume that $k$ changes sign on $\Omega$ and $\int_{\Omega} h=0$. Further assume that $k$ is such that

$$
\int_{\Omega} k e^{u_{0}}<0
$$

where $u_{0}$ satisfies (6.6). Then the problem

$$
\int_{\Omega}[\nabla u \cdot \nabla v+h v]=\int_{\Omega} k e^{u} v, \quad \forall v \in H,
$$

has a solution $u \in H$, i.e., the problem (6.1) subject to the constraints (6.2) has a weak solution.

Proof. Let $u$ be a solution of (6.5) whose existence follows from Corollary 6.1. It follows then from Liusternik's theorem $([\mathrm{K}])$ that there exists $\lambda \in \mathbb{R}$ such that

$$
\int_{\Omega}[\nabla u \cdot \nabla v+h v]=\lambda \int_{\Omega} k e^{u} v, \quad \forall v \in H .
$$

Note that $u \in H^{2}(\Omega)$. In fact, since $\left.u\right|_{\partial \Omega} \equiv \alpha \in \mathbb{R}$, we have that $u_{1}=u-\alpha \in$ $H_{0}^{1}(\Omega)$ and thus (6.8) implies

$$
\begin{aligned}
\int_{\Omega}\left[\nabla u_{1} \cdot \nabla v+h v\right] & =\int_{\Omega} \nabla\left(u_{1}+\alpha\right) \cdot \nabla v+h v \\
& =\lambda \int_{\Omega} k e^{u} v=\lambda \int_{\Omega} k e^{u_{1}+\alpha} v, \quad \forall v \in H_{0}^{1}(\Omega) .
\end{aligned}
$$

Thus $u_{1}$ is a weak solution of

$$
\left\{\begin{array}{l}
\Delta u_{1}+h=\lambda k e^{u_{1}+\alpha}, \quad \text { in } \Omega, \\
u_{1}=0, \quad \text { on } \partial \Omega .
\end{array}\right.
$$

But since $2\left(u_{1}+\alpha\right) \in H^{1}(\Omega)$ we have $\int_{\Omega} e^{2\left(u_{1}+\alpha\right)}<\infty$, i.e., $e^{u_{1}+\alpha} \in L^{2}(\Omega)$, and thus $k e^{u_{1}+\alpha} \in L^{2}(\Omega)$. Since $h \in L^{2}(\Omega)$ we obtain by regularity theory (see, e.g., [GT] ) that $u \in H^{2}(\Omega)$. Hence $w=u_{0}-u \in H^{2}(\Omega)$. This implies that $e^{w} \in H^{1}(\Omega)$. In fact we have (by the above) $e^{w} \in L^{2}(\Omega)$, and, moreover,

$$
\left|\nabla e^{w}\right|=\left|e^{w} \nabla w\right|=e^{w}|\nabla w|
$$

since $|\nabla w| \in L^{2}(\Omega)$, and by the Sobolev embedding theorem, $H^{2}(\Omega) \hookrightarrow$ $L^{\infty}(\Omega)(n=2)$ that $w \in L^{\infty}(\Omega)$, hence $e^{w} \in L^{\infty}(\Omega)$ and therefore $e^{w}|\nabla w| \in$ $L^{2}(\Omega)$; further since $w=u_{0}-u=$ constant on $\partial \Omega$ we get $e^{w} \in H$.

Letting $v=e^{w}=e^{u_{0}-u} \in H$ in (6.9) and (6.6) and subtracting yield

$$
\int_{\Omega} \nabla\left(u-u_{0}\right) \cdot \nabla\left(e^{u_{0}-u}\right)=\lambda \int_{\Omega} k e^{u} e^{u_{0}-u}
$$

or

$$
\int_{\Omega} \nabla\left(u-u_{0}\right) \cdot e^{u_{0}-u} \nabla\left(u_{0}-u\right)=\lambda \int_{\Omega} k e^{u_{0}}
$$

i.e.,

$$
-\int_{\Omega}\left|\nabla\left(u-u_{0}\right)\right|^{2} e^{u_{0}-u}=\lambda \int_{\Omega} k e^{u_{0}}
$$

implying that $\lambda>0$. Putting $\bar{u}=\log \lambda+u$, we obtain a solution of (6.8). 
Remark 6.3. Using similar arguments to the above one may establish the existence of solutions of the problem

$$
\Delta u+k e^{u}=h, \quad \text { in } \Omega
$$

subject to Neumann boundary conditions

$$
\frac{\partial u}{\partial n}=0, \quad \text { on } \partial \Omega,
$$

provided $h$ satisfies, $h \in L^{2}(\Omega)$

$$
\int_{\Omega} h=0
$$

and $k \in C(\bar{\Omega})$ is such that $k$ changes sign and

$$
\int_{\Omega} k e^{u_{0}}<0
$$

where $u_{0}$ satisfies

$$
\int_{\Omega} \nabla u_{0} \cdot \nabla v=\int_{\Omega} h v, \quad \forall v \in H^{1}(\Omega) .
$$

Remark 6.4. The one-dimensional analogue to problem (6.1), (6.2) is the periodic boundary value problem $\left('=\frac{d}{d t}\right)$

$$
\begin{gathered}
u^{\prime \prime}+k e^{u}=h, \quad 0<t<T, \\
u(0)=u(T), u^{\prime}(0)=u^{\prime}(T) .
\end{gathered}
$$

With the proper interpretation Corollary 6.2 represents an existence result for this problem also.

Now, we consider an extension of the example considered above to the case of the $p$-Laplacian. Let $\Omega \subset \mathbb{R}^{N}$ be a bounded domain with smooth boundary and consider the partial differential equation

$$
\nabla \cdot\left(|\nabla u|^{p-2} \nabla u\right)+k e^{u}=h, \quad \text { in } \Omega
$$

subject to the (nonlocal) boundary conditions

$$
\left\{\begin{array}{l}
u \equiv \text { constant, on } \partial \Omega \\
\int_{\partial \Omega} \frac{\partial u}{\partial \nu_{p}}=0,
\end{array}\right.
$$

where $k$ and $h$ are given functions and $\frac{\partial u}{\partial \nu_{p}}=|\nabla u|^{p-2} \frac{\partial u}{\partial \nu}$ is the normal derivative (defined by the $p$-Laplacian) on $\partial \Omega$.

This problem has the weak formulation:

$$
\int_{\Omega}\left[|\nabla u|^{p-2} \nabla u \cdot \nabla v+h v\right]-\int_{\Omega} k e^{u} v=0, \forall v \in V, u \in V,
$$

where

$$
V=\left\{u \in W^{1, p}(\Omega):\left.u\right|_{\partial \Omega}=\text { constant }\right\},
$$

then $V$ is a (Banach) subspace of $W^{1, p}(\Omega)$ with respect to the usual $W^{1, p_{-}}$ norm

$$
\|u\|=\left(\int_{\Omega}\left[|u|^{p}+|\nabla u|^{p}\right]\right)^{1 / p} .
$$


We assume that $p \geq N, k \in C(\bar{\Omega})$, and $h \in L^{q}(\Omega), q=\frac{p}{p-1}$ and define

$$
\begin{gathered}
\varphi(u)=\frac{1}{p} \int_{\Omega}|\nabla u|^{p}, \quad u \in V, \\
j(u)=\int_{\Omega} h u, \quad u \in V,
\end{gathered}
$$

then $j$ is a bounded linear functional, and $\operatorname{ker} \varphi=\mathbb{R}$.

As above, we let

$$
S=\left\{u \in V: \psi(u)=\int_{\Omega} k e^{u}=0\right\},
$$

then $S \neq \varnothing$, whenever $k$ assumes both positive and negative values. Since $p \geq N$, it follows that

$$
\psi: V \rightarrow \mathbb{R}
$$

is a completely continuous mapping. (If $p>N$, this follows from the compact embedding $W^{1, p}(\Omega) \hookrightarrow L^{\infty}(\Omega)$. If $p=N$, as in the case $p=2$, this is an immediate consequence of Trudinger's theorem ([GT]) about the embedding of $W^{1, p}(\Omega)$ into the Orlicz space $L_{\varphi}(\Omega)$ with $\varphi(t)=e^{|t| \frac{N}{N-1}}-1, t \geq 0$ (see also [A]), and compact embedding results between Orlicz spaces ([A]).) Hence $S$ is weakly closed in $V$. Further $\psi \in C^{1}(V, \mathbb{R})$ and

$$
\left\langle\psi^{\prime}(u), v\right\rangle=\int_{\Omega} k e^{u} v, \quad u, v \in V .
$$

Of course $\varphi$ and $j$ are of class $C^{1}$ also and $\varphi^{\prime}+j^{\prime}$ is as computed earlier.

The (quasilinear) variational problem corresponding to $(6.13)$

$$
\int_{\Omega}|\nabla u|^{p-2} \nabla u \cdot \nabla v+h v=0, \forall v \in V, u \in V,
$$

has a solution (as is easily verified) if and only if $\int_{\Omega} h=0$, which we shall assume. We now let $u_{0}$ be a solution of (6.14) (all solutions of which are given by $\left.u_{0}+c, c \in \mathbb{R}\right)$. We have the following existence result.

Corollary 6.5. Assume that $k$ changes sign on $\Omega$ and that $\int_{\Omega} h=0$. Then there exists $u \in S$ such that

$$
F=\varphi(u)+j(u)=\min _{v \in S}[\varphi(v)+j(v)] .
$$

If also

$$
\int_{\Omega} k e^{u_{0}}<0
$$

then (6.13) has a solution.

Proof. We note that $F$ satisfies property $(P)$ by an application of Proposition 4.3 noting that $V=W_{0}^{1, p} \oplus \mathbb{R}, \operatorname{ker} \varphi=\mathbb{R}$. The remaining arguments are parallel to those used in the proof of Corollary 6.2 and Remark 6.3 with the obvious modifications made for the present case. We shall omit the details.

Remark 6.6. Another approach to proving the results of Corollaries 6.1 and 6.5 would be to replace $S$ by $S \cap\left\{u \in V: \int_{\Omega} u=0\right\}$, and proceed in a similar way. 
6.2 Some higher order problems. Let $H=H^{2}(0, T)$, where $T$ is a fixed positive number, equipped with the usual Sobolev norm. Let $a: H \times H \rightarrow \mathbb{R}$ be the bilinear form given by

$$
a(u, v)=\int_{0}^{T} u^{\prime \prime} v^{\prime \prime}, \quad u, v \in H,
$$

and let

$$
\varphi(u)=\frac{1}{2} a(u, u), \quad\langle A u, v\rangle=\frac{1}{2} a(u, v) .
$$

For $\alpha>1, \alpha \neq 2$ and $k \in L^{1}(0, T)$ consider the functional

$$
\psi: H \rightarrow \mathbb{R}
$$

defined by

$$
\psi(u)=\int_{0}^{T} k\left|u^{\prime}\right|^{\alpha} .
$$

It is clear that $a$ is bilinear continuous, symmetric and positive on $H$ (i.e., $a(u, u) \geq 0)$. Since $a(u, u)=0$ implies that $u^{\prime \prime}=0$ a.e. on $(0, T)$, it follows that

$$
\operatorname{ker} a=\{u: u=a t+b, a, b \in \mathbb{R}, t \in[0, T]\} .
$$

Letting $P_{0}=0, P_{1}(v)=\int_{0}^{T}\left[\left(v^{\prime}\right)^{2}+v^{2}\right], v \in H$ we get

$$
a(v, v)+P_{1}(v)=\|v\|^{2}, \quad \forall v \in H .
$$

It therefore follows that $\varphi$ satisfies property $(P)$ on $H$.

On the other hand we have that $\psi \in C^{1}(H, \mathbb{R})$ and

$$
\left\langle\psi^{\prime}(u), v\right\rangle=\alpha \int_{0}^{T} k\left|u^{\prime}\right|^{\alpha-2} u^{\prime} v^{\prime}, \quad u, v \in H .
$$

We shall now use what has been established in section 3 to obtain critical points (nontrivial) of the functional

$$
f: u \mapsto \varphi(u)+\psi(u),
$$

as solutions of the equation

$$
\int_{0}^{T} u^{\prime \prime} v^{\prime \prime}+\alpha \int_{0}^{T} k\left|u^{\prime}\right|^{\alpha-1} u^{\prime} v^{\prime}=0, \quad \forall v \in H^{2}(0, T),
$$

the latter being the weak formulation of the nonlinear boundary value problem

$$
\left\{\begin{array}{l}
u^{(4)}=\alpha\left(k\left|u^{\prime}\right|^{\alpha-2} u^{\prime}\right)^{\prime} \quad \text { in }(0, T), \\
u^{\prime \prime}(0)=u^{\prime \prime}(T)=0, \\
\alpha k(0)\left|u^{\prime}(0)\right|^{\alpha-2} u^{\prime}(0)=u^{\prime \prime \prime}(0), \\
\alpha k(T)\left|u^{\prime}(T)\right|^{\alpha-2} u^{\prime}(T)=u^{\prime \prime \prime}(T) .
\end{array}\right.
$$

It is clear that constants are trivial solutions. To obtain nontrivial solutions we use Corollary 3.3, and obtain 
Corollary 6.7. Assume that $k \in L^{1}(0, T)$ is such that

$$
\int_{0}^{T} k>0 \text { and } \int_{0}^{T} k\left|u^{\prime}\right|^{\alpha}<0, \text { for some } u \in H .
$$

Then (6.21) and hence (6.22) has a nonconstant solution.

Proof. We check the assumptions of Corollary 3.6. By assumption there exists $u \in H$ such that $\psi(u)<0$. Let $u \in \operatorname{ker} \varphi$, then $u(t)=a t+b, a, b \in \mathbb{R}$. Then

$$
\psi(u)=\int_{0}^{T} k\left|u^{\prime}\right|^{\alpha}=\int_{0}^{T} k|a|^{\alpha}=|a|^{\alpha} \int_{0}^{T} k=\left\{\begin{array}{lll}
0, & \text { if } & a=0, \\
>0, & \text { if } & a \neq 0 .
\end{array}\right.
$$

Hence $\psi(u) \geq 0, \forall u \in \operatorname{ker} \varphi$. If $\psi(u)=0$, then $u \equiv b$ and hence $u^{\prime}=0$ and for all $v \in H$, we have

$$
\psi(v-u)=\int_{0}^{T} k\left|v^{\prime}-u^{\prime}\right|^{\alpha}=\int_{0}^{T} k\left|v^{\prime}\right|^{\alpha}=\psi(v) .
$$

We hence may apply Corollary 3.6

Remark 6.8. (a) If $k \in C(0, T) \cap L^{1}(0, T), \int_{0}^{T} k>0$ and $k$ changes sign on $(0, T)$ then the conditions of corollary 6.7 hold.

(b) Since $\operatorname{ker} \varphi \cap \operatorname{ker} \psi \neq\{0\}$ in the above example, Corollary 3.3 is not applicable here.

The next example we consider is similar in nature to the one above but considers a fourth order partial differential equation from the theory of thin elastic plates (see [ASV1] for similar problems subject to unilateral constraints).

Let $\Omega \subset \mathbb{R}^{2}$ be a bounded domain with smooth boundary $\partial \Omega$. Let $H=$ $H^{2}(\Omega)$ (equipped with the usual norm and inner product) and let

$$
a: H \times H \rightarrow \mathbb{R}
$$

be the bilinear form defined by

$$
\begin{array}{r}
a(u, v)=\int_{\Omega}\left[\partial_{11} u \partial_{11} v+\partial_{22} u \partial_{22} v+\nu\left(\partial_{11} u \partial_{22} v+\partial_{22} u \partial_{11} v\right)\right. \\
\left.+2(1-\nu) \partial_{12} u \partial_{12} v\right], \\
0<\nu<\frac{1}{2},
\end{array}
$$

where $\partial_{i j}=\frac{\partial}{\partial x_{i} \partial x_{j}}$.

Then (see [ASV1])

$$
a(u, u) \geq(1-\nu) \int_{\Omega}\left[\left(\partial_{11} u\right)^{2}+\left(\partial_{22} u\right)^{2}+\left(\partial_{12} u\right)^{2}\right],
$$

and thus $a(u, u) \geq 0$. Defining $\varphi(u)=\langle A u, u\rangle$, where again

$$
\langle A u, v\rangle=\frac{1}{2} a(u, v),
$$

we see that the conditions of Proposition 2.3 may be satisfied with $P_{0}=0$ and

$$
P_{1}(u)=\int_{\Omega}|\nabla u|^{2}+\int_{\Omega} u^{2},
$$


and hence that $\varphi$ satisfies property $(P)$. Moreover $a$ is symmetric and

$$
\begin{aligned}
& \text { ker } a=\left\{u \in H^{2}(\Omega): u\left(x_{1}, x_{2}\right)=a x_{1}+b x_{2}+c, \quad a, b, c \in \mathbb{R},\right. \\
& \left.x=\left(x_{1}, x_{2}\right) \in \Omega\right\} \text {. }
\end{aligned}
$$

Let $\psi: H \rightarrow \mathbb{R}$ be given by $\left(k \in L^{\infty}(\Omega)\right)$

$$
\psi(u)=\int_{\Omega} k|\nabla u|^{\alpha}, \quad \alpha>1, \alpha \neq 2 .
$$

Since the embedding $H^{1}(\Omega) \hookrightarrow L^{q}(\Omega)$ is continuous $\forall q \in(1, \infty), \psi$ is well defined. Moreover $\psi \in C^{1}$ and

$$
\left\langle\psi^{\prime}(u), v\right\rangle=\alpha \int_{\Omega} k|\nabla u|^{\alpha-2} \nabla u \cdot \nabla v \quad(u, v \in H) .
$$

Since the embedding $H^{2}(\Omega) \hookrightarrow H^{1}(\Omega)$ is compact we see that $\psi$ is weakly continuous. Further $\psi$ is homogeneous of degree $\alpha$. We obtain the following corollary.

Corollary 6.9. If $\int_{\Omega} k>0$ and $\int_{\Omega} k|\nabla u|^{\alpha}<0$ for some $u \in H^{2}(\Omega)$, then the equation

$$
\left\langle\varphi^{\prime}(u)+\psi^{\prime}(u), v\right\rangle \equiv a(u, v)+\alpha \int_{\Omega} k|\nabla u|^{\alpha-2} \nabla u \cdot \nabla v=0
$$

$\left(\forall v \in H^{2}(\Omega)\right)$ has a nonconstant solution $u \in H^{2}(\Omega)$.

The proof is similar to that of Corollary 6.7. We only note that if $u \in \operatorname{ker} \varphi$, then $u=a x_{1}+b x_{2}+c, \nabla u=(a, b)$ and

$$
\psi(u)=\left(a^{2}+b^{2}\right)^{\alpha / 2} \int_{\Omega} k= \begin{cases}0, & \text { if } a=b=0, \\ >0, & \text { if } a \neq 0 \text { or } b \neq 0 .\end{cases}
$$

Thus $\psi(u) \geq 0$ and $\psi(u)=0$ if and only if $u \equiv$ constant. Thus $\psi(v-u)=$ $\psi(v)$ for all $u \equiv$ constant.

6.3 A semilinear problem involving the $p$-Laplacian. Let $\Omega$ be a bounded domain in $\mathbb{R}^{N}$ with smooth boundary. For $p>1$, consider the boundary value problem

$$
\begin{cases}-\nabla \cdot\left(|\nabla u|^{p-2} \nabla u\right)+g(x, u)=0, & \text { in } \Omega, \\ \frac{\partial u}{\partial \nu_{p}}=|\nabla u|^{p-2} \frac{\partial u}{\partial \nu}=0, & \text { on } \partial \Omega,\end{cases}
$$

where $g: \Omega \times \mathbb{R} \rightarrow \mathbb{R}$ is a given function satisfying Carathéodory type conditions and the growth conditions (given below) (see, e.g., [D], [PM], or [An] and the references therein for usual properties of the $p$-Laplacian).

This problem has the following weak formulation:

$$
\int_{\Omega}|\nabla u|^{p-2} \nabla u \cdot \nabla v+\int_{\Omega} g(x, u) v=0, \forall v \in W^{1, p}(\Omega), u \in W^{1, p}(\Omega),
$$

where $V=W^{1, p}(\Omega)$ is the Sobolev space of $L^{p}$ functions having $L^{p}$ first (weak) derivatives, endowed with the usual norm $\|u\|=\|u\|_{W^{1, p}(\Omega)}$. Concerning $g$ we assume that it is the growth condition

$$
|g(x, u)| \leq a+b|u|^{q-1},
$$


where $a, b$ are constants and

$$
\left\{\begin{array}{l}
1<q<p^{*}=N p(N-p)^{-1}, \text { if } p<N, \\
1<q<p^{*}=\infty, \text { if } N \leq p .
\end{array}\right.
$$

Let

$$
G(x, u)=\int_{0}^{u} g(x, s) d s,
$$

then standard arguments give that solutions of (6.26) are given by the critical points of the functional

$$
f(u)=\varphi(u)+\psi(u),
$$

where

$$
\varphi(u)=\frac{1}{p} \int_{\Omega}|\nabla u|^{p},
$$

and

$$
\psi(u)=\int_{\Omega} G(x, u(x)), \quad u \in V .
$$

It follows that $\varphi$ and $\psi$ are of class $C^{1}$ and

$$
\left\{\begin{array}{l}
\left\langle\varphi^{\prime}(u), v\right\rangle=\int_{\Omega}|\nabla u|^{p-2} \nabla u \cdot \nabla v, \\
\left\langle\psi^{\prime}(u), v\right\rangle=\int_{\Omega} g(x, u(x)) v, \quad \forall u, v \in V,
\end{array}\right.
$$

where $\langle\cdot, \cdot \cdot\rangle$ denotes the pairing between $V$ and $V^{*}$.

With this setup we have the following result.

Corollary 6.10. Assume that

$$
G(x, t u)=t^{\alpha} G(x, u), \forall t \geq 0, \text { a.e. } x \in \Omega, \forall u \in \mathbb{R},
$$

and

$$
\int_{\Omega} G(x, \pm 1)>0
$$

and

$$
\int_{\Omega} G(x, u(x))<0, \text { for some } u \in V .
$$

Then (6.26) has a nontrivial solution.

Proof. We first check that $\varphi$ has property $(P)$. It is clear that $\varphi$ is nonnegative and continuous on $V$ and, since $p>1, \varphi$ is convex and hence weakly lower semicontinuous. We shall employ Proposition 4.2 with $F=\varphi$. Thus let $\left\{v_{n}\right\},\left\{w_{n}\right\}, w$ be as in the proposition. It follows that $\lim _{n \rightarrow \infty} \frac{\varphi\left(v_{n}\right)}{\left\|v_{n}\right\|^{p}}=0$, and hence

$$
\begin{aligned}
0 & \leq \varphi(w) \leq \liminf _{n \rightarrow \infty} \varphi\left(w_{n}\right) \leq \limsup _{n \rightarrow \infty} \varphi\left(w_{n}\right) \\
& =\limsup _{n \rightarrow \infty} \varphi\left(\frac{v_{n}}{\left\|v_{n}\right\|}\right)=\limsup _{n \rightarrow \infty} \frac{\varphi\left(v_{n}\right)}{\left\|v_{n}\right\|^{p}}=0 .
\end{aligned}
$$

Hence $\varphi(w)=0$, i.e., $w=$ constant. Thus also

$$
\int_{\Omega}\left|\nabla\left(w_{n}-w\right)\right|^{p} \rightarrow 0
$$


which together with the compactness of the embedding $V \hookrightarrow L^{p}(\Omega)$ implies that $w_{n} \rightarrow w$. Thus $\varphi$ has property $(P)$.

The above also shows that

$$
\operatorname{ker} \varphi=\mathbb{R}, \varphi(v-u)=\varphi(v), \forall v \in V, \forall u \in \operatorname{ker} \varphi .
$$

The growth conditions on $g$ and (6.27) imply that the embedding $V \hookrightarrow$ $L^{q}(\Omega)$ is compact and thus if $u_{n} \rightarrow u$, then $u_{n} \rightarrow u$ in $L^{q}(\Omega)$ and hence $G\left(\cdot, u_{n}\right) \rightarrow G(\cdot, u)$ in $L^{1}(\Omega)$, which implies the complete continuity of $\psi$. Using the other hypotheses on $g$ we may now employ part (b) of Corollary 5.1 to complete the proof.

A particular choice of $g$ is

$$
g(x, u)=k(x)|u|^{\alpha-2} u,
$$

where $k \in C(\bar{\Omega}), \quad 1<\alpha p^{*}, \alpha \neq p$. In this case the Carathéodory conditions are easily verified. The other conditions of the corollary will hold whenever $k$ changes sign on $\Omega$ and $\int_{\Omega} k>0$.

6.4 A Dirichlet problem involving the $p$-Laplacian. As above let $\Omega$ be a bounded domain in $\mathbb{R}^{N}$ with smooth boundary. We shall now consider a Dirichlet problem, which may be considered as a nonlinear perturbation of a problem at resonance. For $p>1$, consider the boundary value problem

$$
\begin{cases}-\nabla \cdot\left(|\nabla u|^{p-2} \nabla u\right)+\lambda_{1} m(x)|u|^{p-2} u+g(x, u)=0, & \text { in } \Omega, \\ u=0, & \text { on } \partial \Omega,\end{cases}
$$

where $m: \Omega \rightarrow \mathbb{R}, \quad m \in L^{\infty}(\Omega)$ is the weight function with $m>0$ on a set of positive measure, $g$ is as in the above example and $\lambda_{1}$ is the pricipal eigenvalue of the $p$-Laplacian (with respect to the weight $m$ ) with respect to homogeneous Dirichlet boundary data (cf. [An]).

It follows from [An] that $\lambda_{1}$ is a simple eigenvalue, is isolated and can be characterized as

$$
\begin{aligned}
\lambda_{1} & =\inf \left\{\int_{\Omega}|\nabla u|^{p}: u \in W_{0}^{1, p}(\Omega), \int_{\Omega} m|u|^{p}=1\right\} \\
& =\inf \left\{\frac{\int_{\Omega}|\nabla u|^{p}}{\int_{\Omega} m|u|^{p}}: u \in W_{0}^{1, p}(\Omega), \int_{\Omega} m|u|^{p}>0\right\} .
\end{aligned}
$$

Problem (6.32) has the following weak formulation:

$$
\begin{array}{r}
\int_{\Omega}|\nabla u|^{p-2} \nabla u \cdot \nabla v-\lambda_{1} \int_{\Omega} m|u|^{p-2} u+\int_{\Omega} g(x, u) v=0, \\
\forall v \in W_{0}^{1, p}(\Omega), u \in W_{0}^{1, p}(\Omega),
\end{array}
$$

It follows that solutions of (6.34) are given by the critical points of the functional

$$
f(u)=\frac{1}{p} \int_{\Omega}|\nabla u|^{p}-\frac{\lambda_{1}}{p} \int_{\Omega} m|u|^{p}+\int_{\Omega} G(x, u(x)), \quad u \in W_{0}^{1, p}(\Omega) .
$$

We have the following result. ( $\gamma$ is an eigenfunction of the $p$-Laplacian corresponding to $\lambda_{1}$.) 
Corollary 6.11. Assume that

$$
\int_{\Omega} G(x, \pm \gamma)>0
$$

and

$$
\int_{\Omega} G(x, u(x))<0, \text { for some } u \in W_{0}^{1, p}(\Omega) .
$$

Then (6.34) has a nontrivial solution.

Proof. We shall employ the usual norm (implied by the Poincaré inequality) in $V=W_{0}^{1, p}(\Omega)$. Let again $\psi$ be defined by

$$
\psi(u)=\int_{\Omega} G(x, u(x)), \quad u \in V .
$$

It follows that $\psi$ is of class $C^{1}$ and

$$
\left\langle\psi^{\prime}(u), v\right\rangle=\int_{\Omega} g(x, u(x)) v, \forall u, v \in V,
$$

where again $\langle\cdot, \cdot\rangle$ denotes the pairing between $V$ and $V^{*}$. Let

$$
\varphi(u)=\frac{1}{p} \int_{\Omega}|\nabla u|^{p}-\frac{\lambda_{1}}{p} \int_{\Omega} m|u|^{p}, \quad u \in V,
$$

then $\varphi$ is nonnegative. Further, the mapping

$$
u \mapsto \frac{1}{p} \int_{\Omega}|\nabla u|^{p}
$$

is convex and continuous and the mapping

$$
u \mapsto \frac{\lambda_{1}}{p} \int_{\Omega} m|u|^{p}
$$

is completely continuous. Hence the mapping

$$
u \mapsto \varphi(u)
$$

is weakly lower semicontinuous. We next check that $\varphi$ satisfies property $(P)$. We again use Proposition 4.2 with $F=\varphi$. Thus let $\left\{v_{n}\right\},\left\{w_{n}\right\}, w$ be as in the proposition. It follows that $\lim \sup _{n \rightarrow \infty} \frac{\varphi\left(v_{n}\right)}{\left\|v_{n}\right\|^{p}} \leq 0$, and hence

$$
\begin{aligned}
0 & \leq \varphi(w) \leq \liminf _{n \rightarrow \infty} \varphi\left(w_{n}\right) \leq \limsup _{n \rightarrow \infty} \varphi\left(w_{n}\right) \\
& =\limsup _{n \rightarrow \infty} \varphi\left(\frac{v_{n}}{\left\|v_{n}\right\|}\right)=\limsup _{n \rightarrow \infty} \frac{\varphi\left(v_{n}\right)}{\left\|v_{n}\right\|^{p}} \leq 0,
\end{aligned}
$$

i.e., $\varphi(w)=0$, or

$$
\int_{\Omega}|\nabla w|^{p}=\lambda_{1} \int_{\Omega} m|w|^{p},
$$

and

$$
\int_{\Omega}\left|\nabla w_{n}\right|^{p}-\lambda_{1} \int_{\Omega} m\left|w_{n}\right|^{p} \rightarrow 0 .
$$


Since $w_{n} \rightarrow w$ in $V$ it follows that $w_{n} \rightarrow w$ in $L^{p}(\Omega)$ and hence

$$
\lambda_{1} \int_{\Omega} m\left|w_{n}\right|^{p} \rightarrow \lambda_{1} \int_{\Omega} m|w|^{p} .
$$

Therefore (6.39) and (6.40) imply that $w_{n} \rightarrow w$ (recall that $W_{0}^{1, p}(\Omega)$ is uniformly convex). Moreover (6.39) implies that

$$
\operatorname{ker} \varphi=\operatorname{span}\{\gamma\}=\{t \gamma: t \in \mathbb{R}\} .
$$

The remainder of the proof is similar to that of the previous corollary.

\section{REFERENCES}

[A] R. Adams, Sobolev spaces, Academic Press, New York, 1975.

[An] H. Anane, Simplicité et isolation de la première valeur propre du p-Laplacien avec poids, C. R. Acad. Sci. Paris 305 (1987), 725-728.

[ASV1] D. D. Ang, K. Schmitt and L. K. Vy, Noncoercive variational inequalities: some applications, Nonlinear Analysis, TMA 15 (1990), 497-512.

[ASV2] _ Variational inequalities and the contact of elastic plates, Differential Equations and Applications in Biology, Physics, and Engineering (Goldstein, Kappel, Schappacher, eds.), Marcel Dekker, New York, 1991, pp. 1-19.

[ASV3] _ P-coercive variational inequalities and unilateral problems for von Karman's equations, World Scientific Series in Applied Math., vol. 1 (R. Agarwal, ed.), World Scientific, Singapore, 1992, pp. 15-29.

[BTW] A. Ben Naoum, C. Troestler and M. Willem, Existence and multiplicity results for homogeneous second order differential equations, J. Differential Equations (to appear).

[B] H. Brézis, Analyse fonctionelle, Masson, Paris, 1983.

[D] P. Drabek, Solvability and bifurcations of nonlinear equations, Longman Scientific and Technical Series, Essex, 1992.

[GT] D. Gilbarg and N. S. Trudinger, Elliptic partial differential equations of second order, Springer-Verlag, New York, 1983.

[J] R. C. James, Orthogonality and linear functionals in normed spaces, Trans. Amer. Math. Soc. 61 (1947), 265-292.

[KS] D. Kinderlehrer and G. Stampacchia, Introduction to variational inequalities, Academic Press, New York, 1980.

[K] M. A. Krasnosel'skii, Topological methods in the theory of nonlinear integral equations, Pergamon, New York, 1964.

[MW] J. Mawhin and M. Willem, Critical point theory and Hamiltonian systems, Springer, New York, 1989.

[PS] D. Pascali and J. Sburlan, Nonlinear mappings of monotone type, Sijthoff and Noordhoff, Bucharest, 1978.

[PM] M. A. del Pino and R. F. Manasevich, Global bifurcation from the eigenvalues of the p-Laplacian, J. Differential Equations 92 (1991), 226-251.

[S] M. Struwe, Variational methods and their applications in nonlinear partial differential equations, Springer, New York, 1990.

[T] S. L. Trojanski, On locally uniformly convex and differentiable norms in certain nonseparable Banach spaces, Studia Math. 37 (1971), 173-180.

Department of Mathematics, University of Utah, Salt Lake City, Utah 84112 